\title{
Atividade física após o diagnóstico do câncer de mama: Revisão sistemática
}

\author{
Life Physical activity after breast cancer diagnosis: systematic review
}

Leonessa Boing ${ }^{1 *}$, Adriana Coutinho de Azevedo Guimarães ${ }^{1}$, Nycolle Martins Reis ${ }^{1}$, Marina Ribovski $^{1}$

ARTIGO DE REVISÃO | REVIEWARTICLE

\begin{abstract}
Esta revisão sistemática objetivou analisar estudos originais que investigaram o nível de prática de atividade física (AF) autorrelatada, bem como os benefícios e os fatores associados em mulheres em tratamento ou pós-tratamento por câncer de mama (CM). A busca foi realizada nas bases de dados eletrônicas PubMed, Web of Science, Science Direct, OVID e Biblioteca Virtual da Saúde. Utilizaram-se os descritores [physical activity] AND [breast cancer] em inglês, espanhol e português. Foram incluídos 21 estudos publicados em inglês, no período de 2004 a 2014. Em sua maioria, as mulheres não atingiram as recomendações de AF no pós-tratamento, não se encontraram resultados relacionados à prevalência da AF durante o tratamento de CM. Notou-se associação entre nível de prática de AF com faixa etária, IMC, escolaridade, etnia, estágio do $\mathrm{CM}$, menopausa, comorbidades associadas, tabagismo, imagem corporal, sintomas depressivos e presença de fadiga. Maior incidência de AF para caminhada, associada à melhora da qualidade de vida (QV), diminuição do nível de fadiga e de sintomas depressivos.

Palavras-chave: Neoplasias da mama; Atividade Motora; Saúde.
\end{abstract}

ABSTRACT

This systematic review aimed to analyze the original studies that investigated the level of physical activity (PA) self-reported, and the benefits and associated factors among women in treatment or after treatment for breast cancer (BC). In this way, the search was performed in electronic databases PubMed, Web of Science, Science Direct, OVID and Health Virtual Library used the descriptors [physical activity] AND [breast cancer] in English, Spanish and Portuguese. 21 studies published in English were included, between 2004 and 2014. We could find that most of the women did not reached the recommendations of PA posttreatment, there were no findings related to the prevalence of PA during treatment of BC . Noted an association between PA practice level with age, BMI, education, ethnicity, BC stage, menopause, associated comorbidities, smoking, body image, depressive symptoms and presence of fatigue. Higher incidence of PA to walk was associated with improved quality of life (QOL), decreased level of fatigue and depressive symptoms.

Keywords: Breast Neoplasms; Motor Activity; Health.

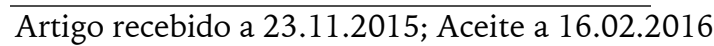

${ }^{1}$ Universidade do Estado de Santa Catarina (UDESC). Florianópolis, Brasil.

* Autor correspondente: Rua Desembargador Pedro Silva, 2034. Bairro: Coqueiros. CEP 88080700. Florianópolis, SC

- Brasil. E-mail: leonessaboing@gmail.com 


\section{INTRODUÇÃO}

Em âmbito mundial, o câncer é considerado um problema de saúde pública que a cada ano ganha maiores proporções. De acordo com a World Health Organization (WHO, 2013) e da International Agency for Research on Cancer (IARC, 2013), a incidência de casos deve aumentar em $57 \%$ nos próximos 20 anos. No Brasil, foram estimados 395 mil novos casos para o ano de 2014, especificamente 57 mil de câncer de mama (CM), o que corresponde a $20,8 \%$ dos novos casos nas mulheres, caracterizando-se como o câncer mais incidente nesta população (Ministério da Saúde do Brasil e Instituto Nacional de Câncer José Alencar Gomes da Silva [INCA], 2014).

O tratamento para o $\mathrm{CM}$, nomeadamente cirurgia (mastectomia radical ou conservadora), quimioterapia, radioterapia ou hormonioterapia, está associado ao comprometimento no organismo destas pacientes, ocasionando consequências físicas, como fadiga e dores (Sabino Neto, Moreira, Resende, \& Ferreira, 2012) além de alterações na imagem corporal, declínios psíquicos, emocionais e sociais (Makluf, Dias, \& Barra, 2006; Soares et al., 2013). Ainda, aponta-se o diagnóstico de sarcopenia atrelado ao declínio físico e até mesmo a mortalidade de pacientes com câncer (Parsons, Baracos, Dhillon, Hong, \& Kurzrock, 2012). Nesse sentido, ressalta-se a necessidade de intervenções que minimizem tais complicações, atrelado à um estilo de vida saudável, principalmente no que diz respeito aos hábitos alimentares e nos níveis de atividade física (AF) (George et al., 2011).

A AF vem sendo associada como uma forma de diminuir os comprometimentos e melhorar a QV destas pacientes (Campos, Hassan, Riechelmann, \& del Giglio, 2011; Costa et al., 2013; Mohammadi, Sulaiman, Koon, Amani, \& Hosseini, 2013; Phillips \& McAuley, 2013; Soares, 2011; Szymlek-Gay, Richards, \& Egan, 2011; Yaw et al., 2014), minimizando os declínios decorrentes do tratamento (Alfano et al., 2007; Basen-Engquist, Hughes, Perkins, Shinn, \& Taylor, 2008; Macêdo et al., 2011; Yaw et al., 2014) e associando-se à uma melhora das capacidades físicas (Soares, 2011). Ainda, em alguns casos são observadas relações positivas com a diminuição do risco de mortalidade após o diagnóstico do CM (Bradshaw et al., 2014; Irwin et al., 2008). Considerando a projeção que a AF vem assumindo na literatura dentro do contexto do CM, objetivou-se por meio de uma revisão sistemática reunir artigos publicados que investigaram a prática de $\mathrm{AF}$ autorrelatada, bem como os benefícios e os fatores associados em mulheres em tratamento ou pós-tratamento do CM.

\section{MÉTODO}

A presente revisão sistemática teve como base os preceitos estabelecidos pelo modelo PRISMA - Preferred Reporting Items for Systematic Reviews and Meta-Analysis (Liberati et al., 2009). Dessa forma, a pergunta de pesquisa formou-se a partir do acrônimo PICOS, caracterizando-se da seguinte maneira: Quais os benefícios da AF autorrelatada em mulheres com CM em tratamento ou póstratamento?

Para busca e seleção dos artigos que respondessem a pergunta de pesquisa, foram selecionadas as principais bases de dados relacionadas à àrea das ciências da saúde, sendo estas: PubMed, Web of Science, Science Direct, OVID e Biblioteca Virtual da Saúde (BVS). A busca aconteceu por meio dos descritores selecionados de acordo com os MeSH terms: [motor activity] AND [breast neoplasms], e seus entryterms [physical activity] OR [locomotor activity] AND [breast cancer] OR [breast tumor] OR [breast carcinoma]. Toda busca eletrônica ocorreu no período de 01 de agosto de 2014 a 01 de setembro de 2014.

Delimitou-se como critérios de inclusão: (a) estudos que foram publicados no período dos anos de 2004 a 2014, (b) nos idiomas inglês, espanhol ou português, (c) caracterizados como estudos transversais, longitudinais, observacionais, epidemiológicos e coorte, (d) que envolvessem os descritores diretamento no título, a fim de garantir que o foco principal dos estudos fosse diretamente associado ao tema da presente revisão sistemática, (e) que incluíssem em sua amostra mulheres com faixa etária a partir dos 18 anos, e por fim (f) que investigassem, apenas por meio de métodos indiretos, especificamente questionários, a 
prática de $\mathrm{AF}$, os benefícios e os fatores associados à prática de $\mathrm{AF}$, em mulheres em tratamento ou pós-tratamento do CM.

Foram definidos como critérios de exclusão: estudos (a) caracterizados metodologicamente como revisões sistemáticas ou da literatura, estudos pilotos, protocolos de estudos, dissertações, teses, capítulos de livros, suplementos ou comentários do editor, (b) que utilizassem o termo exercício ao invés de AF, (c) que empregassem métodos diretos para investigar a AF, uma vez que o foco da presente revisão é identificar a $\mathrm{AF}$ autorrelatada pelas próprias pacientes, (d) que abordassem a $\mathrm{AF}$ como fator de risco para o diagnóstico do $\mathrm{CM}$, pois estes estudos não incluem a $\mathrm{AF}$ após o diagnóstico, e sim no período anterior ao diagnóstico, (e) que investigassem a $\mathrm{AF}$ antes do diagnóstico do $\mathrm{CM}$, ou seja, a AF pregressa ao diagnóstico, (f) que não envolvessem a AF no objetivo principal do estudo, (g) que optassem pela metodologia qualitativa para investigação da $\mathrm{AF}$ e, finalmente, (h) que fossem identificados como referências cruzadas.

Todo o processo de busca nas bases de dados eletrônicos, seleção dos estudos, leitura dos artigos e compilação das informações foi efetuada pela pesquisadora principal do estudo, visando maior confiabilidade do mesmo, e replicado por duas pesquisadoras de maneira cega e independente, sendo que as divergências encontradas foram resolvidas por meio de consenso entre as pesquisadoras. Na primeira etapa do estudo foram identificados os títulos e resumos, além de busca secundária na lista bibliográfica dos artigos. Após exclusão dos artigos que não cumpriram com os critérios de inclusão, foi realizado o fichamento de todos os artigos aptos para leitura integral.

A figura 1 apresenta o fluxograma de estratégia de seleção dos estudos de acordo com as normas PRISMA. Após a busca inicial nas bases de dados, foram encontrados 1417 estudos ao total, sendo detalhadamente 336 no PubMed, 58 na Science Direct, 434 na Web of Science, 391 no BVS e 198 no OVID. Foram retiradas inicialmente 368 referências cruzadas. Seguido pela exclusão de 806 artigos pelos seguintes motivos: 238 por estarem fora da data determinada na metodologia (2004-2014), 76 por caracterizarem-se metodologicamente como revisões sistemáticas ou de literatura, 139 por serem editoriais, suplementos, livros, dissertações ou estudos pilotos, 199 por não apresentarem os descritores no título e no objetivo, 13 por serem em outro idioma, 50 por investigarem o tema em animais, 49 por investigarem o CM no sexo masculino, 259 por não apresentarem os descritores no título ou objetivo principal do estudo, restando dessa forma, 443 artigos elegíveis para leitura integral. Após a leitura integral, 422 artigos foram excluídos pelos seguintes motivos: 172 por tratarem a AF como fator de risco de incidência do CM, 13 por serem de abordagem qualitativa, 42 por investigarem outros tipos de câncer; 34 por utilizarem métodos diretos para análise da AF; 76 por investigarem a AF antes do diagnóstico; 85 por utilizarem o termo exercício. Dessa forma, ao final da seleção foram incluídos fielmente 21 artigos na revisão sistemática.

A qualidade dos dados foi avaliada por meio dos critérios metodológicos propostos por Downs e Black (1998), pontuados em 27 questões de um checklist, que pautava-se em aspectos metodológicos de comunicação, validade externa, validade interna (viés), validade interna (fatores de confusão) e poder estatístico. Para apreciação dos estudos, considerou-se apenas 19 questões, excluindo as questões $4,8,13-15,19,23$ e 24 , por não tratar-se de estudos com intervenções de cunho experimental. Para cada questão, aplicou-se o escore zero, caso o artigo não fosse pertinente ao que se está avaliando, e o escore um (1), quando apresentava resposta positiva ao requisito, sendo que apenas a questão 5 tem escore máximo 2 . Com isso, a pontuação máxima de cada artigo foi de 20 pontos. Esta adaptação foi utilizada em outras revisões sistemáticas, justificando a escolha da mesma (Araujo, Vilarim, Sabroza, \& Nardi, 2010; Schlüssel, Souza, Reichenheim, \& Kac, 2008). A avaliação dos artigos foi realizada por dois pesquisadores da presente revisão, de forma independente. A fim de minimizar eventuais discordâncias na pontuação dos artigos, foi consultada a opinião de um terceiro pesquisador. 
Dessa maneira, os artigos foram avaliados de acordo com os seguintes critérios: a) hipóteses ou objetivos; b) principais desfechos; c) características dos participantes incluídos; d) distribuição das principais variáveis de confusão em cada grupo de sujeitos a ser comparado; e) principais resultados; f) informação sobre estimativas da variabilidade aleatória nos dados para os principais desfechos; g) informação sobre características das perdas; h) informação sobre valores de probabilidade para os principais desfechos; i) representatividade dos indivíduos convidados a participar do estudo; j) representatividade dos indivíduos incluídos no estudo; k) caso os resultados não tenham sido baseados em hipóteses estabelecidas a priori, se isto foi deixado claro; 1) se, em estudos de coorte, a análise ajustou para diferentes durações de acompanhamento, ou, se em estudos de casos e controles o tempo entre a intervenção e o desfecho foi o mesmo para casos e controles; $\mathrm{m}$ ) adequação dos testes estatísticos utilizados para medir os principais desfechos; n) se as medidas utilizadas para os principais desfechos foram acuradas; o) se os participantes em diferentes grupos foram recrutados na mesma população; $p$ ) se os participantes nos diferentes grupos foram recrutados no mesmo período de tempo; q) se a análise incluiu ajuste adequado para as principais variáveis de confusão; r) se foram consideradas as perdas de participantes durante $\mathrm{o}$ acompanhamento; e s) Se o estudo tinha poder suficiente para detectar um efeito importante com um nível de significância de 5\%.

\section{RESULTADOS}

A presente revisão foi composta por 21 artigos.

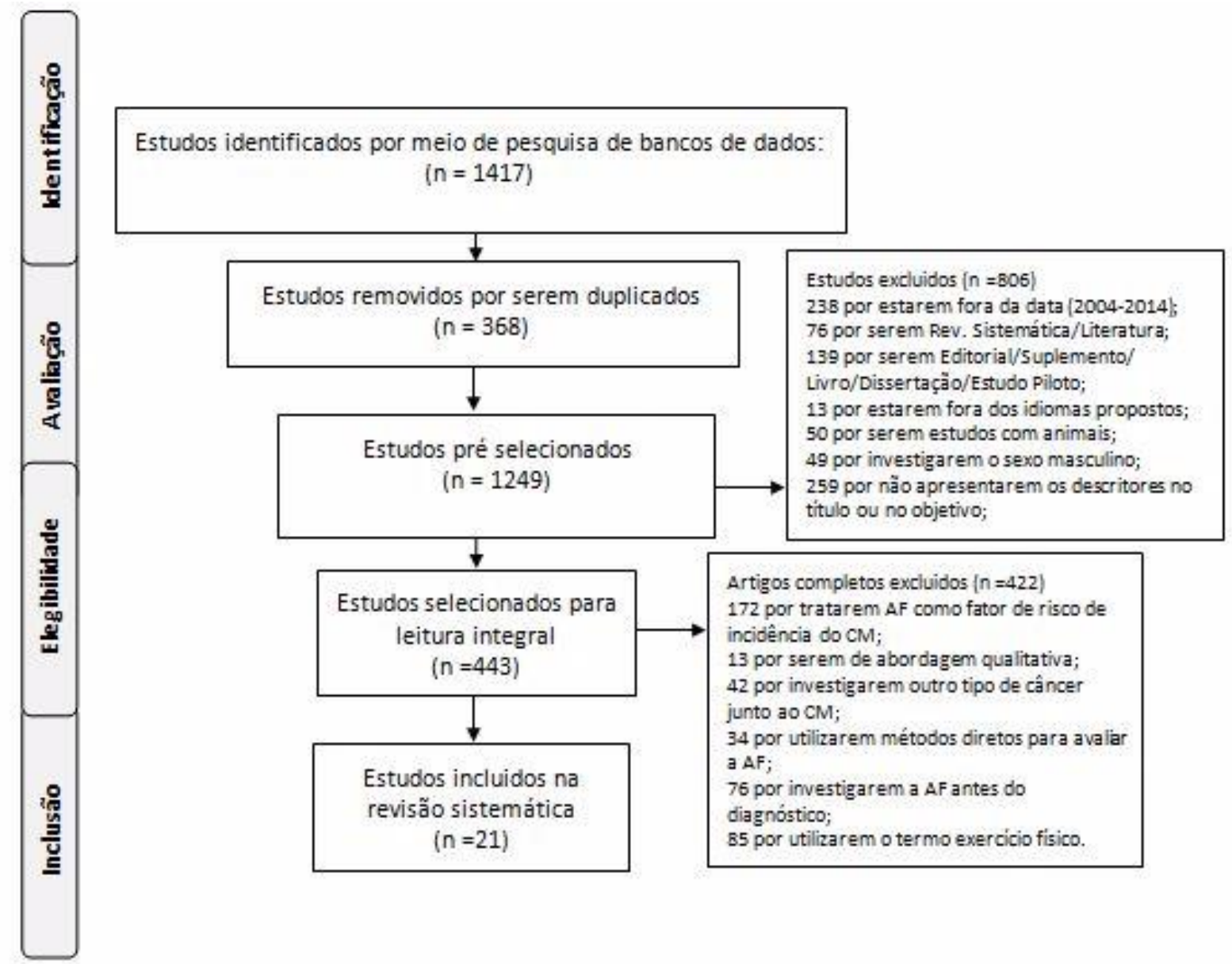

Figura 1. Fluxograma de seleção dos estudos de acordo com o PRISMA. Tabela 1 
159 | L Boing, ACA Guimarães, NM Reis, M Ribovski

Descrição dos artigos selecionados a partir do autor e ano, tipo de estudo, objetivo geral, amostra, instrumentos utilizados e principais resultados.

\begin{tabular}{|c|c|c|c|c|c|c|}
\hline Autor/Ano & $\begin{array}{l}\text { Tipo de } \\
\text { estudo }\end{array}$ & Objetivo & Amostra & $\begin{array}{l}\text { Instrumentos } \\
\text { que avaliaram a } \\
\text { AF }\end{array}$ & Principais resultados & \# \\
\hline $\begin{array}{l}\text { Irwin et al. } \\
(2004)\end{array}$ & $\begin{array}{l}\text { prospectivo de } \\
\text { coorte. }\end{array}$ & $\begin{array}{l}\text { Verificar os níveis de AF nos três anos após o } \\
\text { diagnóstico de } \mathrm{CM} \text { e a influência da obesidade, idade, } \\
\text { estado da doença, bem como a proporção de mulheres } \\
\text { que atingem o nível de AF recomendado. }\end{array}$ & $\begin{array}{l}806 \text { mulheres com } \\
\text { CM (54.9 anos), do } \\
\text { HEAL - Estados } \\
\text { Unidos. } \\
\end{array}$ & AF - MAQ; & $\begin{array}{l}32 \% \text { das mulheres no pós-tratamento de CM atingiram os níveis } \\
\text { recomendados de AF. Menos sobreviventes de CM obesas } \\
\text { atenderam à recomendação, quando comparadas com as em } \\
\text { sobrepeso e magras }(\mathrm{p}<0,005) \text {. }\end{array}$ & 17 \\
\hline $\begin{array}{l}\text { Hong et al. } \\
(2007)\end{array}$ & N.C. & $\begin{array}{l}\text { Analisar os fatores associados à AF de mulheres no pós- } \\
\text { tratamento do CM. }\end{array}$ & $\begin{array}{l}2819 \text { mulheres ( } 27 \\
\text { a } 74 \text { anos) do } \\
\text { WHEL - Estados } \\
\text { Unidos. }\end{array}$ & AF - WHI; & $\begin{array}{l}\text { Características demográficas e variáveis psicossociais foram } \\
\text { associadas com a AF }(\mathrm{p}<0.001) \text {, como etnia, escolaridade, } \\
\text { depressão e IMC. }\end{array}$ & \\
\hline $\begin{array}{l}\text { Basen- } \\
\text { Engquist et al. } \\
\text { (2008) }\end{array}$ & $\begin{array}{l}\text { corte } \\
\text { transversal. }\end{array}$ & $\begin{array}{l}\text { Explorar como as dimensões da AF (gasto energético } \\
\text { total, frequência e duração) são associados com os } \\
\text { sintomas no pós-tratamento do CM. }\end{array}$ & $\begin{array}{l}148 \text { mulheres (>18 } \\
\text { anos) com CM- } \\
\text { Estados Unidos. }\end{array}$ & AF - 7-DPARQ; & $\begin{array}{l}\text { Gasto energético total foi associado com um melhor estado geral de } \\
\text { saúde ( } p=0,006) \text { e menos sintomas depressivos }(p=0,014) \text {, } \\
\text { enquanto a frequência de AF foi linearmente relacionada com o } \\
\text { funcionamento físico }(p=0,047) \text {, dor }(p=0,057) \text {, estado geral de } \\
\text { saúde }(p<0,001) \text {, e sintomas depressivos }(p<0,001) \text {. }\end{array}$ & \\
\hline $\begin{array}{l}\text { Holick et al. } \\
(2008)\end{array}$ & $\begin{array}{l}\text { coorte } \\
\text { prospectivo. }\end{array}$ & $\begin{array}{l}\text { Investigar a relação entre a AF recreacional após o } \\
\text { diagnóstico e o risco de morte em mulheres com CM } \\
\text { invasivo entre } 1988 \text { e } 2001 \text {. }\end{array}$ & $\begin{array}{l}4.482(20 \text { e } 79 \\
\text { anos) mulheres do } \\
\text { CWLS - Estados } \\
\text { Unidos. }\end{array}$ & AF - CWLS; & $\begin{array}{l}\text { Aumentar o nível de } \mathrm{AF} \text { recreacional após o diagnóstico de } \mathrm{CM} \text { pode } \\
\text { aumentar a sobrevida das mulheres com CM. }\end{array}$ & 18 \\
\hline $\begin{array}{l}\text { Smith et al. } \\
(2009)\end{array}$ & obser & $\begin{array}{l}\text { Examinar associações entre AF recrea } \\
\text { sobreviventes de CM em um cor }\end{array}$ & $\begin{array}{l}729 \text { mulheres }(57 \\
\text { anos) do HEAL - } \\
\text { Estados Unidos. }\end{array}$ & $\begin{array}{l}\text { AF recreacional - } \\
\quad \text { MAQ; }\end{array}$ & $\begin{array}{l}\text { Associações entre AF e QV foram encontradas nas mulheres negras } \\
\text { e brancas não hispânicas }(\mathrm{p}<0,05) \text {. Atender as recomendações de } \\
\text { AF foi associado com melhor QV. }\end{array}$ & \\
\hline $\begin{array}{l}\text { Bertram et al. } \\
(2010)\end{array}$ & $\begin{array}{l}\text { randomizado } \\
\text { controlado. }\end{array}$ & $\begin{array}{l}\text { ssociação entre AF no pós-tratamento de } \\
\text { ss adversos ou mortalidade. }\end{array}$ & $\begin{array}{l}2.361 \text { mulheres } \\
\text { com CM do WHEL } \\
\text { - Estados Unidos. }\end{array}$ & $\begin{array}{l}\text { AF - 9-item } \\
\text { WHI; }\end{array}$ & $\begin{array}{l}\text { Mulheres mais ativas tiveram } 57 \% \text { menos chance de mortalidade } \\
\text { quando comparadas com as menos ativas }(\mathrm{p}=0,01) \text {. Aquelas que } \\
\text { mantiveram o nível de AF dentro das recomendações no pós- } \\
\text { tratamento apresentaram diminuição de } 40 \% \text { no risco de } \\
\text { mortalidade. }\end{array}$ & \\
\hline $\begin{array}{l}\text { George et al. } \\
(2011)\end{array}$ & $\begin{array}{l}\text { observacional } \\
\text { de coorte } \\
\text { prospectivo. }\end{array}$ & $\begin{array}{l}\text { mentares e a } \mathrm{AF} \\
\text { prognóstico do }\end{array}$ & $\begin{array}{r}11831 \\
\text { CM } \\
\text { Esta }\end{array}$ & AF - MAQ; & $\begin{array}{l}\text { ativas com melhores hábitos alimentares têm redução de } \\
\text { isco de mortalidade por qualquer causa (IC: } 0.04,0.36 \text { ) e } \\
\text { le } 91 \% \text { de mortalidade por CM (IC: } 0.01,0.89 \text { ). }\end{array}$ & \\
\hline $\begin{array}{l}\text { Malicka et al. } \\
(2011)\end{array}$ & $\begin{array}{l}\text { Estudo de } \\
\text { levantamento } \\
\text { de diagnósticos } \\
\text { (survey). }\end{array}$ & $\begin{array}{l}\text { Avaliar o impacto de diferentes tipos de AF sobre a } \\
\text { satisfação com a vida, a aceitação da doença e adaptação } \\
\text { ao CM entre as mulheres no pós-tratamento. }\end{array}$ & $\begin{array}{l}36 \text { mulheres (59 } \\
\text { anos) com CM - } \\
\text { Polônia. }\end{array}$ & $\begin{array}{l}\text { AF - questões } \\
\text { específicas; }\end{array}$ & $\begin{array}{l}94 \% \text { das mulheres afirmaram ser necessária a prática de } \mathrm{AF} \text {, com } \\
\text { maior frequência do relato da prática de dança; aquelas que } \\
\text { envolveram-se em mais de cinco tipos de } \mathrm{AF} \text { apresentaram maiores } \\
\text { condições de enfrentamento da doença }(\mathrm{p}=0.019) \text {. Falta de tempo } \\
\text { como barreira para a prática. }\end{array}$ & 10 \\
\hline $\begin{array}{l}\text { Mandelblatt } \\
\text { et al. (2011) }\end{array}$ & $\begin{array}{l}\text { corte } \\
\text { transversal. }\end{array}$ & $\begin{array}{l}\text { Examinar as associações entre os níveis de AF e QV em } \\
\text { pacientes durante o tratamento do CM. }\end{array}$ & $\begin{array}{l}2.279 \text { mulheres } \\
\text { com CM - KPNC - } \\
\text { Estados Unidos. }\end{array}$ & AF - AAFQ; & $\begin{array}{l}\text { Mulheres que relataram níveis mais altos de AF apresentaram } \\
\text { maior } \mathrm{QV}(\mathrm{p}<0.0001) \text {; mulheres mais jovens eram mais ativas } \\
\text { durante o tratamento }(\mathrm{p}<0.0001) \text {. }\end{array}$ & \\
\hline $\begin{array}{l}\text { Rogers et al. } \\
(2011)\end{array}$ & $\begin{array}{l}\text { corte } \\
\text { transversal. }\end{array}$ & $\begin{array}{l}\text { Descrever padrões de AF e associações com fadiga e } \\
\text { sintomas depressivos entre mulheres com CM no pós- } \\
\text { tratamento, residentes de cidades rurais. }\end{array}$ & $\begin{array}{l}483 \text { mulheres }(63 \\
\text { anos) com CM- } \\
\text { Estados Unidos. }\end{array}$ & $\begin{array}{l}\text { AF - IPAQ versão } \\
\text { longa; }\end{array}$ & $\begin{array}{l}\text { Idade mais avançada foi associado com menor nível de prática; } \mathrm{AF} \\
\text { foi associada com a fadiga nos domínios doméstico }(\mathrm{p}=.0019) \text {; } \\
\text { lazer }(\mathrm{p}=.047) \text {; com a intensidade moderada }(\mathrm{p}=.011) \text { e com o } \\
\text { tempo sentado }(\mathrm{p}=.0029) \text {. E com a depressão no domínio do lazer } \\
(\mathrm{p}=.039) \text {. }\end{array}$ & \\
\hline $\begin{array}{l}\text { Beasley et al. } \\
\text { (2011) }\end{array}$ & epidemiológico. & $\begin{array}{l}\text { Investigar se a recomendação de AF ( } 2,5 \text { horas/semana } \\
\text { de AF moderada) pode ser estendida para sobreviventes } \\
\text { de CM. }\end{array}$ & $\begin{array}{l}13.302 \text { mulheres } \\
\text { com CM do LACE; } \\
\text { NHS; SBCSS e } \\
\text { WHEL - Estados } \\
\text { Unidos e China. }\end{array}$ & $\begin{array}{l}\text { AF - } \\
\text { Questionários } \\
\text { dos estudos: } \\
\text { LACE; NHS; } \\
\text { SBCSS; WHEL. }\end{array}$ & $\begin{array}{l}\text { As que atingiram as recomendações eram mais jovens, tinham } \\
\text { doença em fase inicial, menos propensas a fumar, com peso normal. } \\
\text { Atingir as recomendações foi associado com redução de } 27 \% \text { na } \\
\text { mortalidade por qualquer causa (IC: } 0.66-0.82 \text { ), e não houve } \\
\text { associação com a recorrência da doença. }\end{array}$ & \\
\hline
\end{tabular}


Charlier et al. corte Avaliar a associação entre sintomas do tratamento, 464 mulheres com

(2012) transversal. social e estratégias de doping na AF no pós-tratamento.

Descrever a mudança nos níveis de AF autorrelatada no

Kwan et al. coorte

(2012) prospectivo fatores associados com essas possíveis mudanças.

\section{Bélgica.}

AF - FPAQ

1.696 mulheres com KPNC Estados Unidos. 3013 mulheres (18 Unidos.

Identificar os comportamentos associados com a 69 mulheres no mudança de comportamento da AF em mulheres idosas pós-tratamento do com CM, e examinar a interrelação entre processo de $\mathrm{CM}$ (71.5 anos) comportamento, autoconfiança, e comportamento em Estados Unidos.

Cardin N.C. relação à AF.

Mohammadi corte et al. (2013) transversal.

Determinar associação entre hábitos alimentares 100 mulheres com saudáveis, AF e a QV de mulheres no pós-tratamento do CM (32 a 61 anos)CM.
(2012) fatores psicológicos, representação da doença, suporte CM (18 a 65 anos) início do diagnóstico e 6 meses depois, e determinar

Paxton et al. randomizado Investigar as associações entre QV, IMC e níveis de AF a 70 anos) com CM (2012) controlado. em mulheres após o CM. do WHEL - Estados

Loprinzi e

O controle pessoal, os sintomas do tratamento (dores no braço e fadiga) e variáveis psicológicas (imagem corporal e depressão) 16 foram associadas à prática de AF no pós-tratamento.

Houve uma redução na prática de AF moderada a vigorosa no diagnóstico e seis meses após $(\mathrm{p}<0.0001)$. Estar na quimioterapia e acima do peso são preditores fortes de declínio da AF moderada a vigorosa.

Atingir as recomendações associou-se à maiores índices de saúde

AF - WHI; física $(p<0.05)$, vitalidade $(p<0.05)$, qualidade de vida relacionada a saúde $(\mathrm{p}<0.05)$ e saúde geral $(\mathrm{p}<0.05)$. Obesidade apresentou maior impacto sobre a saúde física de hispânicos e brancos.

A maioria das pacientes não utiliza todos os processos comportamentais, e a autoconfiança é imprescindível na associação com a A. Os profssionais da saúde tem papel definitivo no encorajamento da aderência à prática de $\mathrm{AF}$ em mulheres idosas com CM.

As mulheres com melhores hábitos alimentares tiverem melhor QV. $65 \%$ relataram a prática de $\mathrm{AF}$ de baixa intensidade. AF apresentou correlação com a QV na função emocional ( $\mathrm{p}=0.004)$ e cognitiva $(\mathrm{p}=0.03)$

$69 \%$ das pacientes são ativas mais de uma vez por semana por pelo menos $\geq 30$ minutos, com fatores associados: maior idade, menor

342 mulheres

Investigar quais $\mathrm{AF}$ as pacientes com $\mathrm{CM}$ inicial
praticam ou tem interesse em praticar, e quais assuntos

Templeton et corte relacionados gostariam de receber maiores informações. sobreviventes ao $\mathrm{CM}$ em estágio inicial (29 a 94 anos) - Suíça.

Questionário ansiedade, e uso de CAM. 87\% reportaram dar atenção aos hábitos 15 próprio sobre AF; alimentares; $46 \%$ utilizam CAM, em sua maioria utilizando vitaminas ou chás. E 45\% modificaram seu estilo de vida após o diagnóstico do $\mathrm{CM}$.

1423 mulheres (25 AF recreacional -

Bradshaw et base Investigar fatores associados com a sobrevivência após a 91 anos) com CM al. (2014) populacional. o diagnóstico de CM.

do LIBCSP -

Questionário

modificado

As mulheres que praticaram $\mathrm{AF}>9.0 \mathrm{METh} / \mathrm{sem}$ apresentaram 17

Estados Unidos.

Determinar a associação entre os sintomas 300 mulheres (61.5 musculoesqueléticos associados a inibição aromatase anos) pós

Brown et al. corte

(2014) transversal. subgrupo para analisar as limitações funcionais associada com redução na AF.

CM - Estados Unidos.

Identificar a trajetória da AF em mulheres com CM em 199 mulheres (55 período de 1 ano após tratamento, e avaliar fatores que anos) com $\mathrm{CM}$ podem predizer essa trajetória.

Canadá.

AF - Questões

iniço da inibição de aromatase. Inibidores de aromatase foram associados com sintomas musculoesqueléticos $(\mathrm{p}=0.001)$ na redução da AF.

Mulheres que reportaram maiores preocupações com o CM maior nível de fadiga mais inativas. A trajetória de AF após o 15

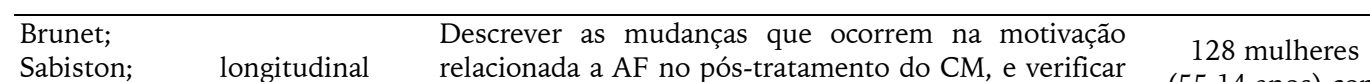

Gaudreau prospectivo. as associações entre os fatores específicos do CM e o (55.14 anos) com apresentaram-se mais ativas, e aquelas com sintomas depressivos e maior hivel de fadiga mais inativas. A trajetoria de AF apos o tratamento não se caracteriza como uniforme no estudo citado. Os processos de auto-apresentação mantiveram-se estáveis, mas o

AF - LTEQ;

nível de AF modificou-se com o decorrer do tempo. Radioterapia

foi associada com a motivação e a construção da impressão pessoal,

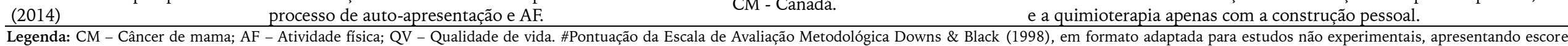

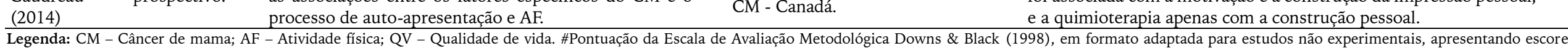

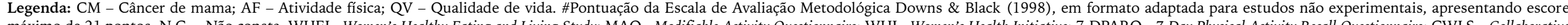

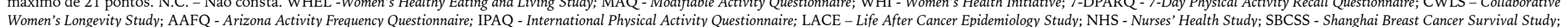
FPAQ - Flemish Physical Activity Questionnaire; CHAMPS - Community Healthy Activities Model Program for Seniors; LIBCSP - Long Island Breast Cancer Study Project; LTEQ - Leisure Time Exercise Questionnaire. 
Todos os artigos selecionados foram publicados em língua inglesa entre os anos de 2004 e 2014, sendo que o ano de 2011 obteve o maior número de publicações, totalizando cinco artigos. O periódico mais frequente foi o Breast Cancer Research And Treatment, com cinco artigos incluídos, e a base de dados predominante foi a Pubmed $(n=15)$, seguida pela Bireme $(n=14)$ e Web of Science $(n=14)$, considerando que um mesmo artigo pode ter sido encontrado em duas ou mais bases de dados, caracterizando-se como referência cruzada.

$\mathrm{Na}$ tabela 1, encontram-se as informações detalhadas de cada artigo. O tipo de estudo foi relatado em 19 artigos selecionados, com predomínio dos estudos de corte transversal $(n=7)$, seguido pelos estudos de coorte prospectivo $(\mathrm{n}=5)$.

A amostra total de mulheres participantes nos estudos, nas diferentes faixas etárias, foi de 36362, com variação de 36 a 13302 pacientes, todas diagnosticadas com CM. Em todos os casos as pacientes ainda estavam em tratamento para o $\mathrm{CM}$, ou já haviam finalizado, encontrando-se no período de pós-tratamento ou sobrevida, termos utilizados nos estudos. A AF esteve relacionada em todos os estudos com outra variável, como $\mathrm{QV}$, avaliações psicossociais, fadiga, fatores metabólicos, hábitos alimentares, depressão, com destaque para a associação entre a AF e a $\mathrm{QV}$, presente em seis artigos.

Em relação aos instrumentos, todos os artigos foram condizentes com o critério de inclusão da utilização de métodos indiretos, ou seja, medidas subjetivas por meio do uso do questionário. Identificaram-se diferentes questionários empregados para avaliação da AF, sendo estes Modifiable Activity Questionnaire (MAQ); International Physical Activity Questionnaire (IPAQ); 7-Day Physical Activity Recall Questionnaire (7DPARQ); Arizona Activity Frequency Questionnaire (AAFQ) e Flemish Physical Activity Questionnaire (FPAQ).

No que se refere às pontuações obtidas por meio da Escala Metodológica Downs e Black (1998), os artigos obtiveram média de 16.04, considerando a pontuação máxima de 20 pontos. Dentre os critérios metodológicos que mais apresentaram falhas, alude-se à omissão das características dos pacientes perdidos durante o seguimento do estudo, à falta de definição do embasamento dos resultados (análises não elucidadas a priori e não detalhamento das distribuições dos principais fatores de confusão), aspectos estes relacionados ao poder de comunicação e validade interna (viés) dos estudos.

\section{DISCUSSÃO}

A partir da observação dos 21 estudos selecionados nesta revisão sistemática, apenas quatro (Basen-Engquist et al., 2008; Brunet, Amireault, et al., 2014; Irwin et al., 2004; Smith et al., 2009) apontaram a prevalência de mulheres ativas no pós-tratamento do CM. Considerando como ativas aquelas que atingiram as recomendações de AF para pacientes com câncer propostas pelo American College of Sports Medicine (ACSM, 2010), o que corresponde à frequência de cinco vezes por semana de 30 minutos de AF moderada ( $150 \mathrm{~min} / \mathrm{sem}$ ), ou três vezes de 25 minutos de AF vigorosa (75min/sem). No estudo de Brunet, Amireault, Chauton, e Sabiston (2014), 49\% das mulheres no período de um ano no pós-tratamento do CM foram consideradas ativas, seguidas por $41 \%$ daquelas no período de cinco anos de póstratamento de Basen-Engquist, Hughes, Perkins, Shinn, e Taylor (2008), 34\% das mulheres no pós-tratamento de dois anos e meio de Smith et al. (2009), e $32 \%$ das mulheres no póstratamento de três anos do estudo de Irwin et al. (2004). Diante disso, observa-se uma oscilação de $49 \%$ a $32 \%$ de prevalência de mulheres ativas nas amostras entre um até cinco anos após finalização do tratamento do CM.

Esses índices merecem atenção, uma vez que atingir as recomendações de AF moderada a vigorosa está associado à benefícios para a saúde destas mulheres, com efeitos positivos na qualidade de vida (Hong et al., 2007; Malicka, Szczepańska-Gieracha, Jankowska, Woź niewski, \& Rymaszewska, 2011; Mandelblatt et al., 2011; Paxton et al., 2012; Smith et al., 2009), emocional, cognitivo (Mohammadi et al., 2013), e diretamente na vitalidade (Paxton et al., 2012). Da mesma forma, acentua-se que a AF resulta em uma melhora da capacidade funcional (Basen- 
Engquist et al., 2008), diminuição da depressão e fadiga (Basen-Engquist et al., 2008; Rogers et al., 2011), das dores associadas (Basen-Engquist et al., 2008) e menores casos de ansiedade (Malicka et al., 2011).

É importante destacar que os resultados dos estudos selecionados apresentaram apenas a prevalência das mulheres no pós-tratamento, revelando a lacuna para estudos que investiguem a AF no período do tratamento clínico, e em suas diferentes categorias: quimioterapia, radioterapia e hormonoterapia. Nesse sentido, salientam-se cinco estudos que citaram a AF relacionada ao tratamento (Brown et al., 2014; Charlier et al., 2012; Hong et al., 2007; Kwan et al., 2012; Mandelblatt et al., 2011). Em que apesar de não apresentarem valores de prevalência, encontraram associações entre os tipos de tratamento e a prática de AF. Detalhadamente, no estudo de Hong et al. (2007), as pacientes que passaram pelo tratamento quimioterápico apresentaram menores níveis de AF no período do pós-tratamento, quando comparadas àquelas que receberam o tratamento radioterápico. Assim como no estudo de Charlier et al. (2012), onde investigaram as mulheres três semanas a seis meses após finalização do tratamento, e apresentaram que aquelas que passaram pela quimioterapia relataram menores níveis de prática de $\mathrm{AF}$ moderada a vigorosa. Em relação à reduções na prática decorrente do tratamento, no estudo de Kwan et al. (2012), as mulheres foram investigadas em dois momentos, dois meses e seis meses após o diagnóstico, apontando maiores reduções no nível de AF moderada a vigorosa, àquelas pacientes que estavam apenas na quimioterapia, quando comparadas às que estavam na quimioterapia e radioterapia concomitantemente, com redução de 2 horas e 15 minutos por semana. Revela-se o tratamento da quimioterapia como preditor de reduções na prática de $\mathrm{AF}$ das mulheres com $\mathrm{CM}$.

Averigou-se também a relação da hormonioterapia com a prática de $\mathrm{AF}$, em que as mulheres que utilizavam inibidores de aromatase eram menos ativas comparadas às que faziam uso do tamoxifeno (Mandelblatt et al., 2011). Além disso, foram relatadas diminuições no nível de AF após início do uso dos inibidores de aromatase, processo justificado pelos sintomas musculoesqueléticos específicos consequentes desse tipo de tratamento, com declínios observados na função física dos membros inferiores, dos braços e dos ombros (Brown et al., 2014). Observa-se que o tratamento do CM, é um fator que contribui para a diminuição da prática de AF de forma geral. No entanto, é necessário evidenciar que a prática de AF neste período está associada com uma melhor qualidade de vida (Mandelblatt et al., 2011), ressaltando-se a importância do estímulo à prática durante o período do tratamento (Hong et al., 2007; Kwan et al., 2012).

Além dos tipos de tratamento, outros fatores também são determinantes na prática de $\mathrm{AF}$ autorrelatada das mulheres com CM, como a etnia (Mandelblatt et al., 2011; Smith et al., 2009), na qual mulheres negras foram as que menos atingiram as recomendações, comparadas com hispânicas e brancas; a escolaridade, revelando que um maior nível de escolaridade está associado à um maior nível de prática (Bertram et al., 2010; Holick et al., 2008; Hong et al., 2007; Rogers et al., 2011); o status de peso, em que o aumento do IMC associou-se com o menor nível de AF (Beasley et al., 2011; Bertram et al., 2010; Brown et al., 2014; Holick et al., 2008; Hong et al., 2007; Irwin et al., 2004) e; idade, quando as mulheres de maior faixa etária demonstraram menor prática de AF (Beasley et al., 2011; Bertram et al., 2010; Brown et al., 2014; Holick et al., 2008; Hong et al., 2007; Irwin et al., 2004). Nesta última questão relacionada à idade, constatam-se divergências por meio do estudo de Bertram et al. (2010), no qual mulheres mais velhas são relatadas como mais ativas no póstratamento quando comparadas as mais novas. Tais discordâncias podem ser provenientes das diferentes formas de seleção da amostra e das amplas faixas etárias selecionadas para os estudos.

Aspectos associados ao quadro da doença foram semelhantemente apontados como fatores que podem predizer menores níveis de AF, nomeadamente estágios avançados do câncer (Holick et al., 2008; Kwan et al., 2012), serem menopausadas no diagnóstico (Bertram et al., 2010; Kwan et al., 2012), menor ocorrência de 
uso do tabaco (Beasley et al., 2011; Bertram et al., 2010), maior presença de comorbidades associadas (Rogers et al., 2011), pior imagem corporal, sintomas no braço (Charlier et al., 2012), sintomas de depressão e a presença de fadiga (Brunet, Sabiston, et al., 2014; Charlier et al., 2012; Hong et al., 2007; Rogers et al., 2011). Além destes, as mulheres no pós-tratamento relataram a falta de tempo como empecilho para prática (Malicka et al., 2011), bem como, a falta de incentivo dos profissionais da área da saúde, que tem papel definitivo no encorajamento da aderência à prática de AF (Loprinzi \& Cardinal, 2013).

No que se refere ao tipo de AF autorrelatada pelas mulheres no pós-tratamento do $\mathrm{CM}$ houve destaque para a caminhada (Irwin et al., 2004; Malicka et al., 2011) e para a dança (Malicka et al., 2011). O estudo de Malicka, SzczepańskaGieracha, Jankowska, Woźniewski, e Rymaszewska (2011) de forma geral, aborda outras atividades que podem ser desenvolvidas com as mulheres no pós-tratamento do $\mathrm{CM}$, como exercícios gerais, andar de bicicleta, exercícios na água e natação. Não foram encontrados relatos do tipo de AF durante o tratamento do $\mathrm{CM}$, sugerindo-se estudos que investiguem essa questão.

Também não foram encontradas convergências entre os questionários nos estudos selecionados. Observa-se que em alguns casos, os dados são provenientes de projetos específicos como Health, Eating, Activity, and Lifestyle Study (HEAL) (George et al., 2011; Smith et al., 2009), Women's Health Initiative (Bertram et al., 2010; Hong et al., 2007; Paxton et al., 2012), Women's Longevity Study (CWLS) (Holick et al., 2008), Community Healthy Activities Model Program for Seniors (CHAMPS) (Loprinzi \& Cardinal, 2013) e Long Island Breast Cancer Study Project (LIBCSP) (Bradshaw et al., 2014), todos os projetos citados tiveram seu desenvolvimento nos Estados Unidos. O estudo de Beasley et al. (2011) com o After Breast Cancer Pooling Project (ABCPP), reúne quatro estudos epidemiológicos, nomeadamente o Life After Cancer Epidemiology Study (LACE); o Nurses' Health Study (NHS), o Shanghai Breast Cancer Survival Study (SBCSS) e o Women's Healthy Eating and Living Study (WHEL), e utiliza apenas as questões de AF recreacional dos estudos citados.

Foram utilizados também questionários validados como Modifiable Activity Questionnaire (George et al., 2011; Irwin et al., 2004; Smith et al., 2009), o 7-Day Physical Activity Recall Questionnaire (7-DPARQ) (Basen-Engquist et al., 2008), O International Physical Activity Questionnaire (IPAQ) na forma longa (Rogers et al., 2011) e na curta (Mohammadi et al., 2013), o Arizona Activity Frequency Questionnaire (Kwan et al., 2012; Mandelblatt et al., 2011) e o Flemish Physical Activity Questionnaire (FPAQ) (Charlier et al., 2012). E ainda, questões desenvolvidas pelos próprios pesquisadores (Brown et al., 2014; Malicka et al., 2011). As limitações relacionadas ao uso de questionários de autorrelato são indicadas em onze estudos (Bertram et al., 2010; Bradshaw et al., 2014; Brown et al., 2014; Brunet, Sabiston, et al., 2014; Charlier et al., 2012; George et al., 2011; Kwan et al., 2012; Loprinzi \& Cardinal, 2013; Mandelblatt et al., 2011; Paxton et al., 2012; Templeton et al., 2013). Evidencia-se ainda a validação dos questionários com o uso de acelerômetros (Basen-Engquist et al., 2008; Bertram et al., 2010) em apenas dois dos estudos selecionados. O que comprova a necessidade de questionários validados especificamente para a população com câncer.

Uma das limitações do presente estudo, pode ser o fato de não ter sido controlada a atividade física anterior ao diagnóstico, e nem terem sido feitas comparações entre as mulheres com câncer de mama e sem a doença, a fim de comprovar que a diminuição do nível de atividade física no período posterior ao câncer está realmente associada à presença da doença ou a outros fatores, como envelhecimento ou comorbidades associadas à idade.

As mulheres com $\mathrm{CM}$ relataram mudanças positivas no seu estilo de vida após o diagnóstico, destacando-se os hábitos alimentares e prática de AF (Templeton et al., 2013). Conferindo ao período de diagnóstico de $\mathrm{CM}$ um caráter de aprendizado, possibilitando a adoção de comportamentos saudáveis a fim de melhorar a qualidade de vida, por meio da prática de AF e dos hábitos alimentares (Brunet, Amireault, et al., 2014). Dessa forma, elucida-se a necessidade 
de criação de programas de intervenção de $\mathrm{AF}$ para mulheres com CM que busquem permear os benefícios da prática e propiciar melhoras na qualidade de vida e na recuperação da capacidade funcional e da rotina diária (Basen-Engquist et al., 2008; Templeton et al., 2013).

\section{CONCLUSÃO}

Analisando os estudos que fizeram parte desta revisão no aspecto AF e CM, observa-se que a maioria das mulheres no pós-tratamento não atingem as recomendações de AF. Destaca-se a importância da AF na melhora da qualidade de vida destas mulheres, amenizando as consequências do tratamento e alterando os aspectos psicológicos que afetam o processo de retorno à vida cotidiana no pós-tratamento.

Diante do contexto, recomendam-se novos estudos a fim de investigar a AF em diferentes momentos do tratamento e pós-tratamento, com designs longitudinais (Mandelblatt et al., 2011) e randomizados (Basen-Engquist et al., 2008). Por fim, ao observar a divergência entre os instrumentos e suas formas de avaliação, tornase indispensável o desenvolvimento de instrumentos subjetivos específicos de avaliação da AF para pacientes com câncer.

Agradecimentos:

Nada a declarar

Conflito de Interesses:

Nada a declarar.

Financiamento:

Nada a declarar

\section{REFERÊNCIAS}

Alfano, C. M., Smith, A. W., Irwin, M. L., Bowen, D. J., Sorensen, B., Reeve, B. B., ... McTiernan, A. (2007). Physical activity, long-term symptoms, and physical health-related quality of life among breast cancer survivors: A prospective analysis. Journal of Cancer Survivorship, 1(2), 116. http://doi.org/10.1007/s11764-007-0014-1

American College of Sports Medicine - ACSM. (2010). ACSM's Resource Manual for Guidelines for Exercise Testing and Prescription (6. ${ }^{\mathrm{a}}$ ed.). Philadelphia:
Wolters Kluwer Health/Lippincott Williams \& Wilkins.

Araujo, D. M. R., Vilarim, M. M., Sabroza, A. R., \& Nardi, A. E. (2010). Depressão no período gestacional e baixo peso ao nascer: uma revisão sistemática da literatura. Cadernos de Saúde Pública, 26(2), 219-227. http://doi.org/10.1590/S0102311X2010000200002

Basen-Engquist, K., Hughes, D., Perkins, H., Shinn, E., \& Taylor, C. C. (2008). Dimensions of physical activity and their relationship to physical and emotional symptoms in breast cancer survivors. Journal of Cancer Survivorship, 2(4), 253-261. http://doi.org/10.1007/s11764-008-0067-9

Beasley, J. M., Kwan, M. L., Chen, W. Y., Weltzien, E. K., Kroenke, C. H., Lu, W., ... Caan, B. J. (2011). Meeting the physical activity guidelines and survival after breast cancer: findings from the after breast cancer pooling project. Breast Cancer Research and Treatment, 131(2), 637-643. http://doi.org/10.1007/s10549-011-1770-1

Bertram, L. A. C., Stefanick, M. L., Saquib, N., Natarajan, L., Patterson, R. E., Bardwell, W., ... Pierce, J. P. (2010). Physical activity, additional breast cancer events, and mortality among earlystage breast cancer survivors: findings from the WHEL Study. Cancer Causes \& Control, 22(3), 427-435. http://doi.org/10.1007/s10552-0109714-3

Bradshaw, P. T., Ibrahim, J. G., Khankari, N., Cleveland, R. J., Abrahamson, P. E., Stevens, J., ... Gammon, M. D. (2014). Post-diagnosis physical activity and survival after breast cancer diagnosis: the Long Island Breast Cancer Study. Breast Cancer Research and Treatment, 145(3), 735742. http://doi.org/10.1007/s10549-014-2966-y

Brown, J. C., Mao, J. J., Stricker, C., Hwang, W.-T., Tan, K.-S., \& Schmitz, K. H. (2014). Aromatase Inhibitor Associated Musculoskeletal Symptoms are associated with Reduced Physical Activity among Breast Cancer Survivors. The Breast Journal, 20(1), 22-28. http://doi.org/10.1111/tbj.12202

Brunet, J., Amireault, S., Chaiton, M., \& Sabiston, C. M. (2014). Identification and prediction of physical activity trajectories in women treated for breast cancer. Annals of Epidemiology, 24(11), 837842.

http://doi.org/10.1016/j.annepidem.2014.07.00 4

Brunet, J., Sabiston, C. M., \& Gaudreau, P. (2014). A prospective investigation of the relationships between self-presentation processes and physical activity in women treated for breast cancer. Health Psychology: Official Journal of the Division of Health Psychology, American Psychological Association, 33(3), 205-213. http://doi.org/10.1037/a0030414

Campos, M. P. de O., Hassan, B. J., Riechelmann, R., \& del Giglio, A. (2011). Fadiga relacionada ao câncer: uma revisão. Revista da Associação Médica 
Brasileira, $\quad 57(2)$,

211-219.

http://doi.org/10.1590/S0104-

42302011000200021

Charlier, C., Van Hoof, E., Pauwels, E., Lechner, L., Spittaels, H., Bourgois, J., \& De Bourdeaudhuij, I. (2012). Treatment-related and psychosocial variables in explaining physical activity in women three weeks to six months post-treatment of breast cancer. Patient Education and Counseling, $89(1)$, 171-177. http://doi.org/10.1016/j.pec.2012.06.029

Costa, F., Silva, B., Galvão, T. S., Lourenço, J. de L., Pereira, A. C. P., \& Bergmann, A. (2013). Atividade física e funcionalidade em mulheres submetidas a tratamento cirúrgico para o câncer de mama após 10 anos de seguimento. Cadernos UNISUAM de Pesquisa e Extensão, 3(1), 84-85.

Downs, S. H., \& Black, N. (1998). The feasibility of creating a checklist for the assessment of the methodological quality both of randomised and non-randomised studies of health care interventions. Journal of Epidemiology and Community Health, 52(6), 377-384.

George, S. M., Irwin, M. L., Smith, A. W., Neuhouser, M. L., Reedy, J., McTiernan, A., ... BallardBarbash, R. (2011). Postdiagnosis diet quality, the combination of diet quality and recreational physical activity, and prognosis after early-stage breast cancer. Cancer Causes \& Control, 22(4), 589-598. http://doi.org/10.1007/s10552-0119732-9

Holick, C. N., Newcomb, P. A., Trentham-Dietz, A., Titus-Ernstoff, L., Bersch, A. J., Stampfer, M. J., ... Willett, W. C. (2008). Physical activity and survival after diagnosis of invasive breast cancer. Cancer Epidemiology, Biomarkers \& Prevention: A Publication of the American Association for Cancer Research, Cosponsored by the American Society of Preventive Oncology, 17(2), 379-386. http://doi.org/10.1158/1055-9965.EPI-07-0771

Hong, S., Bardwell, W. A., Natarajan, L., Flatt, S. W., Rock, C. L., Newman, V. A., ... Pierce, J. P. (2007). Correlates of physical activity level in breast cancer survivors participating in the Women's Healthy Eating and Living (WHEL) Study. Breast Cancer Research and Treatment, 101(2), 225-232. http://doi.org/10.1007/s10549-006-9284-y

International Agency for Research on Cancer. (2013). Latest world cancer statistics Global cancer burden rises to 14.1 million new cases in 2012: Marked increase in breast cancers must be addressed. Press release, $\mathrm{n}^{\circ}$ 223. Obtido de https://www.iarc.fr/en/mediacentre/pr/2013/pdfs/pr223_E.pdf

Irwin, M. L., McTiernan, A., Bernstein, L., Gilliland, F. D., Baumgartner, R., Baumgartner, K., \& BallardBarbash, R. (2004). Physical activity levels among breast cancer survivors. Medicine and Science in Sports and Exercise, 36(9), 1484-1491.

Irwin, M. L., Smith, A. W., McTiernan, A., BallardBarbash, R., Cronin, K., Gilliland, F. D., ...
Bernstein, L. (2008). Influence of pre- and postdiagnosis physical activity on mortality in breast cancer survivors: the health, eating, activity, and lifestyle study. Journal of Clinical Oncology: Official Journal of the American Society of Clinical Oncology, 26(24), 3958-3964. http://doi.org/10.1200/JCO.2007.15.9822

Kwan, M. L., Sternfeld, B., Ergas, I. J., Timperi, A. W., Roh, J. M., Hong, C.-C., ... Kushi, L. H. (2012). Change in physical activity during active treatment in a prospective study of breast cancer survivors. Breast Cancer Research and Treatment, $131(2)$, 679-690. http://doi.org/10.1007/s10549-011-1788-4

Liberati, A., Altman, D. G., Tetzlaff, J., Mulrow, C., Gøtzsche, P. C., Ioannidis, J. P. A., ... Moher, D. (2009). The PRISMA statement for reporting systematic reviews and meta-analyses of studies that evaluate healthcare interventions: explanation and elaboration. BMJ (Clinical Research Ed.), 339, b2700.

Loprinzi, P. D., \& Cardinal, B. J. (2013). Self-efficacy mediates the relationship between behavioral processes of change and physical activity in older breast cancer survivors. Breast Cancer (Tokyo, Japan), 20(1), 47-52. http://doi.org/10.1007/s12282-011-0298-x

Macêdo, G. D. de, Lucena, N. M. G. de, Soares, L. M. de M. M., Rocha, P. O. A. da, Gutièrrez, C. V., \& López, M. C. B. (2011). Influência do estilo de vida na qualidade de vida de mulheres com câncer de mama. Revista Brasileira de Ciências da Saúde, 14(4), 13-18.

Makluf, A. S. D., Dias, R. C., \& Barra, A. A. (2006). Avaliação da qualidade de vida em mulheres com câncer de mama. Revista Brasileira de Cancerologia, 52(1), 49-58.

Malicka, I., Szczepańska-Gieracha, J., Jankowska, E., Woźniewski, M., \& Rymaszewska, J. (2011). Physical activity, life satisfaction and adjustment to illness in women after treatment of breast cancer. Wspótczesna Onkologia, 15(3), 180-185. http://doi.org/10.5114/wo.2011.23011

Mandelblatt, J. S., Luta, G., Kwan, M. L., Makgoeng, S. B., Ergas, I. J., Roh, J. M., ... Kushi, L. H. (2011). Associations of physical activity with quality of life and functional ability in breast cancer patients during active adjuvant treatment: the Pathways Study. Breast Cancer Research and Treatment, 129(2), 521-529. http://doi.org/10.1007/s10549-011-1483-5

Ministério da Saúde do Brasil, \& Instituto Nacional de Câncer José Alencar Gomes da Silva [INCA]. (2014). Estimativa 2014: Incidência de Câncer no Brasil (Coordenação de Prevenção e Vigilância). Rio de Janeiro: Instituto Nacional de Câncer José Alencar Gomes da Silva/ Ministério da Saúde. Obtido

de www.inca.gov.br/estimativa/2014/index.asp?ID $=2$

Mohammadi, S., Sulaiman, S., Koon, P. B., Amani, R., \& Hosseini, S. M. (2013). Impact of healthy 
eating practices and physical activity on quality of life among breast cancer survivors. Asian Pacific Journal of Cancer Prevention: APJCP, 14(1), 481487.

Parsons, H. A., Baracos, V. E., Dhillon, N., Hong, D. S., \& Kurzrock, R. (2012). Body composition, symptoms, and survival in advanced cancer patients referred to a phase I service. PloS One, $7(1)$,

e29330. http://doi.org/10.1371/journal.pone.0029330

Paxton, R. J., Phillips, K. L., Jones, L. A., Chang, S., Taylor, W. C., Courneya, K. S., \& Pierce, J. P. (2012). Associations among physical activity, body mass index, and health-related quality of life by race/ethnicity in a diverse sample of breast cancer survivors. Cancer, 118(16), 4024-4031. http://doi.org/10.1002/cncr.27389

Phillips, S. M., \& McAuley, E. (2013). Physical activity and fatigue in breast cancer survivors: a panel model examining the role of self-efficacy and depression. Cancer Epidemiology, Biomarkers $\mathcal{E}$ Prevention: A Publication of the American Association for Cancer Research, Cosponsored by the American Society of Preventive Oncology, 22(5), 773-781. http://doi.org/10.1158/1055-9965.EPI-12-0983

Rogers, L. Q., Markwell, S. J., Courneya, K. S., McAuley, E., \& Verhulst, S. (2011). Physical activity type and intensity among rural breast cancer survivors: patterns and associations with fatigue and depressive symptoms. Journal of Cancer Survivorship: Research and Practice, 5(1), 54 61. http://doi.org/10.1007/s11764-010-0160-8

Sabino Neto, M., Moreira, J. R., Resende, V., \& Ferreira, L. M. (2012). Physical activity in women undergoing mastectomy and breast reconstruction. Revista Brasileira de Cirurgia Plástica, 27(4), 556-561. http://doi.org/10.1590/S198351752012000400015

Schlüssel, M. M., Souza, E. B. de, Reichenheim, M. E., \& Kac, G. (2008). Physical activity during pregnancy and maternal-child health outcomes: a systematic literature review. Cadernos De Saúde Pública, 24(Suppl 4), s531-544.
Smith, A. W., Alfano, C. M., Reeve, B. B., Irwin, M. L., Bernstein, L., Baumgartner, K., ... BallardBarbash, R. (2009). Race/ethnicity, physical activity, and quality of life in breast cancer survivors. Cancer Epidemiology, Biomarkers $\mathcal{E}$ Prevention: A Publication of the American Association for Cancer Research, Cosponsored by the American Society of Preventive Oncology, 18(2), 656-663. http://doi.org/10.1158/1055-9965.EPI-08-0352

Soares, P. B. M., Carneiro, J. A., Rocha, L. A., Gonçalves, R. C. R., Martelli, D. R. B., Silveira, M. F., \& Martelli Júnior, H. (2013). The quality of life of disease-free Brazilian breast cancer survivors. Revista Da Escola De Enfermagem Da U S $P, 47(1), 69-75$. http://doi.org/10.1590/s008062342013000100009

Soares, W. T. E. (2011). Parameters, considerations and modulation of programs physical exercise for patient oncologic: a systematic review. Revista Brasileira de Medicina do Esporte, 17(4), 284-289. http://doi.org/10.1590/S151786922011000400015

Szymlek-Gay, E. A., Richards, R., \& Egan, R. (2011). Physical activity among cancer survivors: a literature review. The New Zealand Medical Journal, 124(1337), 77-89.

Templeton, A. J., Thürlimann, B., Baumann, M., Mark, M., Stoll, S., Schwizer, M., ... Ruhstaller, T. (2013). Cross-sectional study of self-reported physical activity, eating habits and use of complementary medicine in breast cancer survivors. BMC Cancer, 13, 153. http://doi.org/10.1186/1471-2407-13-153

World Health Organization [WHO]. (2013). Global Health Estimates: Breast Cancer. World Health Organization. Obtido de www.who.int/healthinfo/global_burden_disease /en/

Yaw, Y. H., Shariff, Z. M., Kandiah, M., Weay, Y. H., Saibul, N., Sariman, S., \& Hashim, Z. (2014). Diet and physical activity in relation to weight change among breast cancer patients. Asian Pacific Journal of Cancer Prevention: APJCP, 15(1), 39-44.

Todo o conteúdo da revista Motricidade está licenciado sob a Creative Commons, exceto quando especificado em contrário e nos conteúdos retirados de outras fontes bibliográficas 